\title{
Individuals with ventromedial frontal damage have more unstable but still fundamentally transitive preferences
}

Authors: Linda Q. Yu ${ }^{1,2}$, Jason Dana ${ }^{3}$ and Joseph W. Kable ${ }^{1}$

${ }^{1}$ Department of Psychology, University of Pennsylvania, Philadelphia, PA, 19104

${ }^{2}$ Department of Neuroscience, Brown University, Providence, RI, 02912

${ }^{3}$ Yale School of Management, Yale University, New Haven, CT, 06520

\section{Corresponding author:}

Linda Q. Yu

Carney Institute for Brain Science

Box 1901

Brown University

Providence, RI, 02912

Phone: 401- 863-9057

linda_yu@brown.edu 


\begin{abstract}
The ventromedial frontal lobes (VMF) are important for decision-making, but the precise causal role of the VMF in the decision process has not yet fully been established. Previous studies have suggested that individuals with VMF damage violate transitivity, a hallmark axiom of rational decisions. However, these prior studies cannot properly distinguish whether individuals with VMF damage are truly prone to choosing irrationally from whether their preferences are simply more variable. We had individuals with focal VMF damage, individuals with other frontal damage, and healthy controls make repeated choices across three categories artwork, chocolate bar brands, and gambles. Using sophisticated tests of transitivity, we find that, without exception, individuals with VMF damage make rational decisions consistent with transitive preferences, even though they exhibit greater variability in their preferences. That is, the VMF is necessary for having strong and reliable preferences, but not for being a rational decision maker. VMF damage affects the noisiness with which value is assessed, but not the consistency with which value is sought.
\end{abstract}

Keywords: transitivity, lesion, ventromedial prefrontal cortex, decision-making 


\section{Introduction}

A central assumption of rational choice theories is that decision-makers compare the subjective value of different options and choose the highest valued option. Satisfying this assumption is equivalent to having transitive preferences (1). An example of transitivity is the following: If you prefer to listen to Adele (A) over Beyoncé (B), and Beyoncé over Celine Dion (C), then you would also prefer Adele (A) over Celine (C). There is a strong argument that preferences ought to be transitive, as an intransitive chooser would get caught in choice cycles that do not advance towards any goal (choosing A over B, B over C, and C over A) and that could be exploited (e.g., an unsavory ticket hawker can keep charging you to trade for tickets to your ever-shifting more preferred artist). Thus, nearly all normative theories of decision-making are transitive.

Given this, one might expect that organisms to have internal representations of value that are transitive. Indeed, dozens of functional neuroimaging studies in humans and neurophysiological studies in non-human animals have now identified neural activity in the ventromedial frontal lobe (VMF) that scales with subjective value across different categories of goods (2-5). Correspondingly, lesions to the VMF impair value-based decision making in a variety of ways (6-10).

However, the precise role of the VMF in transitive preference and rational choice is still unclear. Intriguingly, several previous studies have shown that individuals with VMF damage make more cyclical choices (i.e., choosing $\mathrm{C}$ over A after previously selecting A over B and B over C) than healthy controls or individuals with damage elsewhere in the frontal lobe (11-13). However, this increase in cyclical choices after VMF damage is consistent with two very 
different possibilities regarding the necessary role of VMF, and putative value signals in VMF, in transitive preference and rational choice.

The first possibility is that the preferences of individuals with VMF damage are fundamentally intransitive and not self-consistent. In this case, in the example above, the individual with VMF damage would consistently and reliably choose $\mathrm{C}$ over A. This could occur if individuals with VMF damage choose according to stimulus-response associations or rules that lack any higher order transitive structure, rather than according to any set of underlying preferences. If this possibility were true, the proper conclusion would be that an intact VMF is necessary for the human brain to assess value at all, or to use value to make decisions; that an intact VMF is necessary for transitive preferences and rational choice.

The second possibility is that the preferences of individuals with VMF damage are transitive and self-consistent, but are more noisy. In this case, in the example above, the individual with VMF damage might prefer A over $\mathrm{C}$ on average, but less decisively than others. Thus, if asked again later, this individual would have a greater chance of changing their mind and now choosing $\mathrm{C}$ over $\mathrm{A}$. This could occur if individuals with VMF damage choose according to underlying values, but the assessment of those values was noisier or more variable across time. If this possibility were true, the proper conclusion would be that an intact VMF promotes the stability and reduces the variability of valuations across time; but an intact VMF is not necessary for transitive preferences and rational choice.

Distinguishing between preferences that are fundamentally intransitive and preferences that are simply more variable is a deep problem in testing theories of rational choice that has only recently been solved by Regenwetter and colleagues (14). Importantly, these authors recognize that behavior in experiments is probabilistic, and therefore testing axioms of rational 
choice like transitivity requires recasting these axioms in probabilistic terms (14-17). Critically, from a probabilistic perspective, noting a choice cycle (e.g., choosing C over A when one has chosen A over B and B over C), as done in previous studies, is not sufficient to disentangle whether one has fundamentally intransitive versus more variable preferences. Here we use these recently developed tests of a stochastic model of transitivity to determine whether the preferences of individuals with VMF damage are more variable or fundamentally intransitive. If the choices of individuals with VMF damage do not satisfy tests of a stochastic model of transitivity, this would show that their preferences are fundamentally intransitive; alternatively, if their choices do satisfy such tests, this would suggest that their preferences are simply more variable. A definitive answer to this question will identify more precisely the necessary role of VMF, and putative value signals in VMF, in value-based decision-making.

\section{Results}

We tested thirteen individuals with VMF damage, and for comparison, ten individuals with damage to frontal lobe (including dorsomedial, dorsolateral, and insular areas) other than VMF (referred to as frontal controls, or FC), and twenty age and education-matched healthy controls (HC; see Methods for inclusion criteria). Figure 1 shows the overlap of lesions in the two lesion groups, and Table 1 provides the demographic characteristics of all three groups. 


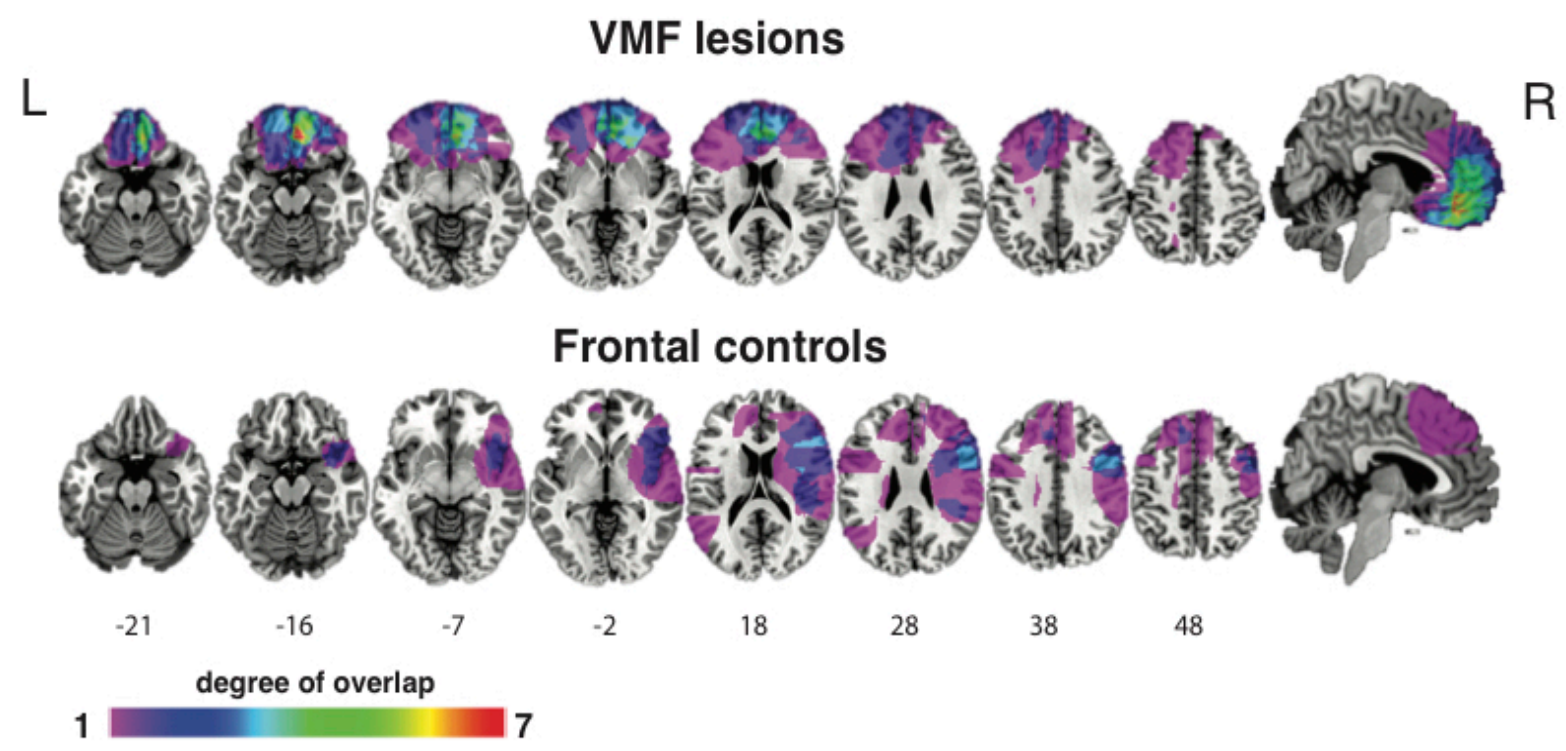

Figure 1. Overlap of lesions in the VMF and frontal control lesion groups. Numbers below slices indicate the MNI z-coordinates. Colors indicate extent of overlap. $\mathrm{L}=1 \mathrm{eft}$; $\mathrm{R}=$ right.

Participants made binary preference decisions in three categories: artworks, chocolate bar brands, and monetary gambles. Each category had two sets of options. Set A, used for stochastic tests of transitivity, contained 5 items that were used to construct 10 binary choices that were each repeated 15 times throughout the experiment. Set B, used to test for choice cycles, contained 10 or 11 items (10 for chocolate bar brands, 11 for art and gambles) that were used to construct 45 or 55 binary choices that were each presented once. Choices using set A and set B items were intermingled in each block. All items in the artwork and chocolate bar brand sets were normed to be as close in value as possible, and all gambles were of equal expected value (see Methods for details). 


\section{Almost all individuals, and all individuals with VMF damage, make choices consistent with stochastic models of transitivity}

Our central question is whether or not the choices of individuals with VMF damage violate stochastic models of transitivity. If they do, this would show that the preferences of individuals with VMF damage are fundamentally intransitive; if they do not, this would suggest that their preferences might more variable, but are not intransitive.

To answer this question, we examined the subset of choices in our experiment, Set A, which involve 15 repetitions each of 10 different binary choices in each of the three categories. In each category, we can calculate 10 choice percentages, one for each of the possible pairings of five items. Table 2 shows these choice percentages in each category for four example participants with VMF damage. Two things are notable about these choice percentages. First, most percentages are less than $100 \%$, demonstrating the need for a probabilistic model of choice. Second, most percentages are greater than 50\%, suggesting that choices are nonetheless systematic in some way and not completely random.

These data provide sufficient power for evaluating whether the choices each participant made are consistent with a stochastic model of transitive choice called the mixture model. The mixture model, developed by Regenwetter and colleagues (14), assumes that every choice is made according to a preference ordering, but that the preference ordering governing a specific choice is drawn randomly from a mixture of all possible preference orderings. Regenwetter and colleagues (14) show that these assumptions place a constraint on the observable choice percentages called the triangle inequalities (see Methods) and develop novel statistical methods for determining when violations of the triangle inequalities are unlikely to be due to random sampling. Given five stimuli and ten pairwise choice percentages, the mixture model imposes 
rather restrictive constraints; only $5 \%$ of all possible choice percentages satisfy the triangle inequalities.

The results of evaluating the mixture model were unambiguous: across nearly all individuals tested, including those with VMF damage, choices were consistent with this stochastic model of transitivity (127 of 129 total tests across all individuals and domains, Table 3). None of the individuals with VMF damage violated the mixture model in any of the three domains (a total of 39 tests, Table 3). Similarly, none of the FC individuals violated the mixture model in any of the three domains (a total of 30 tests, Table 3). Only two HC individuals significantly violated the mixture model, and only in the gambles domain $(p=0.002$ and $p=$ 0.01, respectively, Table 3). Interestingly, both of these individuals followed Tversky (15)'s lexicographic semiorder heuristic, and so their results demonstrate the sensitivity of our test to detect individuals using attribute-based heuristics that lack higher order transitive structure.

\section{Individuals with VMF damage have more variable preferences}

We find that the choices of individuals with VMF damage are consistent with stochastic models of transitivity. This suggests that the previously documented tendency of these individuals to exhibit more choice cycles may be due to having preferences that are more variable, rather than preferences that are intransitive. That is, their choices reflect underlying transitive preference orderings, but they vacillate among preference orderings more than other choosers. However, an alternative explanation is that the individuals that we tested with VMF damage do not exhibit the same alterations in decision-making that have been documented previously in other individuals with VMF damage. To rule out this alternative explanation, we 
performed two additional analyses to directly show that the preferences of individuals with VMF damage in our study are more variable.

First, we fit each individual's choices and response times (RTs) to a drift diffusion model (DDM) that allowed us to directly estimate the variability, or noise, in their preferences (18). We fit the DDM to the same choices used to test the mixture model. The DDM assumes that choices and RTs are a probabilistic function of the rank distance between the two options in the average preference ordering for that individual (see Methods for details on how the average preference ordering was determined). Specifically, we modelled the decision process as resulting from a decision variable (DV), which starts at an initial value (int) that is constant across trials, and then, after a period of non-decision time $(n d t)$, increases linearly across time with a slope $d^{*} v^{\alpha}$, where $d$ is the drift rate, $v$ is the rank difference between the two items, and $\alpha$ is an exponent accounting for potential non-linearities in the effect of rank difference. At each time step, there is also Gaussian noise with a standard deviation of $\varepsilon$ added to the DV, which is the key parameter of interest in our case (see Fig. 2a for a graphical illustration of this model). A decision is made when the DV crosses a fixed threshold value corresponding to a choice of the higher or lower ranked item.

Fits to this model revealed that individuals with VMF damage were more variable choosers. We conducted a 2-way mixed measures ANOVA of the number of choice cycles made by each participant, with item Category (art, brands, gambles) as a within-subject factor and Group (VMF, FC, or HC) as a between-subjects factor. The only parameter of the DDM that was significantly different across groups was the noise parameter $\varepsilon[\mathrm{F}(2,37)=6.25, p=0.005]$. Specifically, the VMF group (mean $=0.12, \mathrm{sd}=0.03$ ) had significantly higher $\varepsilon$ than $\mathrm{HC}$ (mean $=0.09, \mathrm{sd}=0.04)[\mathrm{t}(28)=2.08, p=0.047]$ and $\mathrm{FC}($ mean $=0.07, \mathrm{sd}=0.02)[\mathrm{t}(20)=3.94, \mathrm{p}<$ 
$0.001]$. No other parameters differed between the three groups [drift rate, $d: \mathrm{F}(2,37)=1.78, p=$ 0.18 ; non-linearity, $\alpha: \mathrm{F}(2,37)=0.06, p=0.64$; initial value, int: $\mathrm{F}(2,37)=0.24, p=0.79$; nondecision time, $n d t: \mathrm{F}(2,37)=0.02, p=0.94$; Figure $2 \mathbf{b}]$. The complete set of results from the ANOVAs on the parameters are reported in the Supplementary Materials.

A model-free examination of RTs further supports the conclusion that individuals with VMF damage differ from healthy controls specifically in the noise parameter in the DDM (Figure 2c). An increase in the noise parameter in the DDM causes faster RTs both for choices consistent with the average preference ranking (i.e., "correct" choices) and for choices inconsistent with the average preference ranking (i.e., "errors"). In contrast, an increase in the drift rate in the DDM only speeds "correct" choices. Indicative of an increase in noise, individuals with VMF damage exhibited numerically, though not significantly, faster RTs for “correct" choices $(\mathrm{VMF}$ mean correct $\mathrm{RT}=1.86 \mathrm{~s}, \mathrm{sd}=0.43 ; \mathrm{HC}$ mean correct $\mathrm{RT}=2.32 \mathrm{~s}, \mathrm{sd}=$ $0.90 ; \mathrm{t}(28)=1.3, p=0.35)$ and significantly faster RTs for "error" choices compared to HCs. $(\mathrm{VMF}$ mean error $\mathrm{RT}=4.82 \mathrm{~s}, \mathrm{sd}=0.91 ; \mathrm{HC}$ mean error $\mathrm{RT}=6.37 \mathrm{~s}, \mathrm{sd}=2.10 ; \mathrm{t}(28)=2.29, p=$ $0.03)$. 


\section{A.}

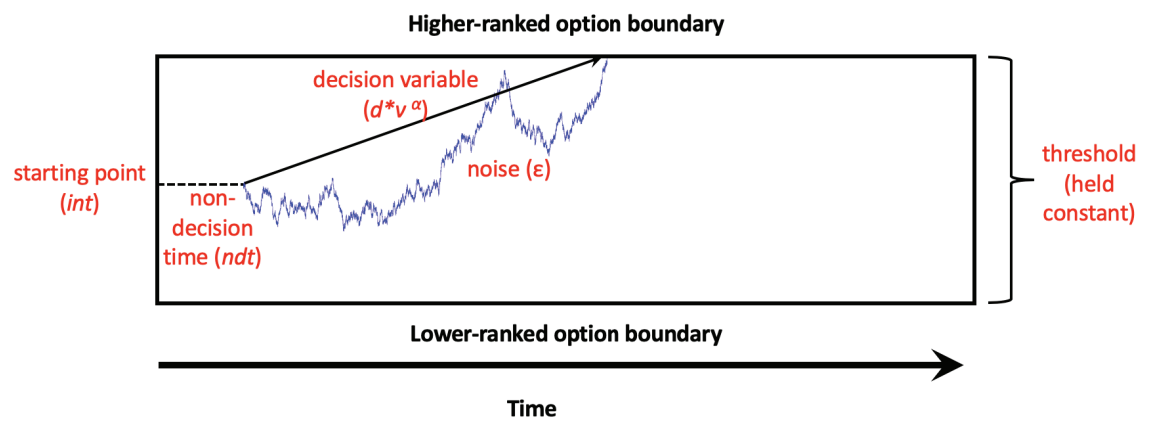

B.
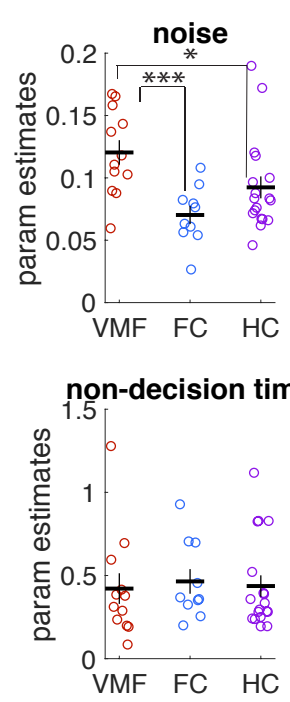
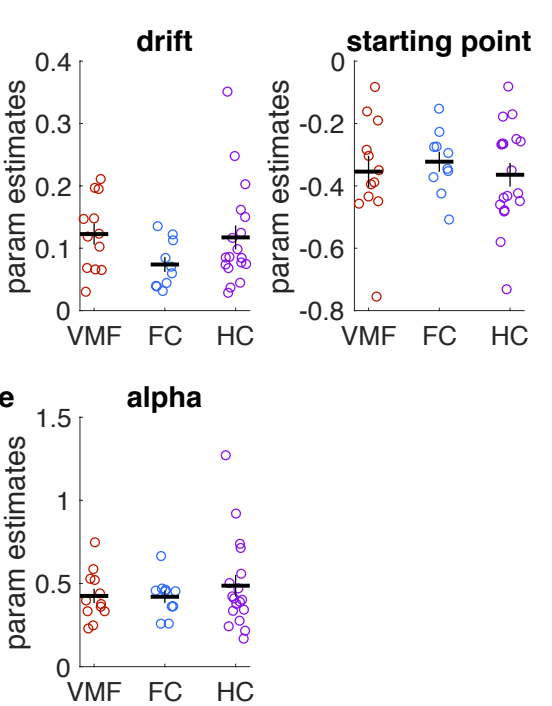
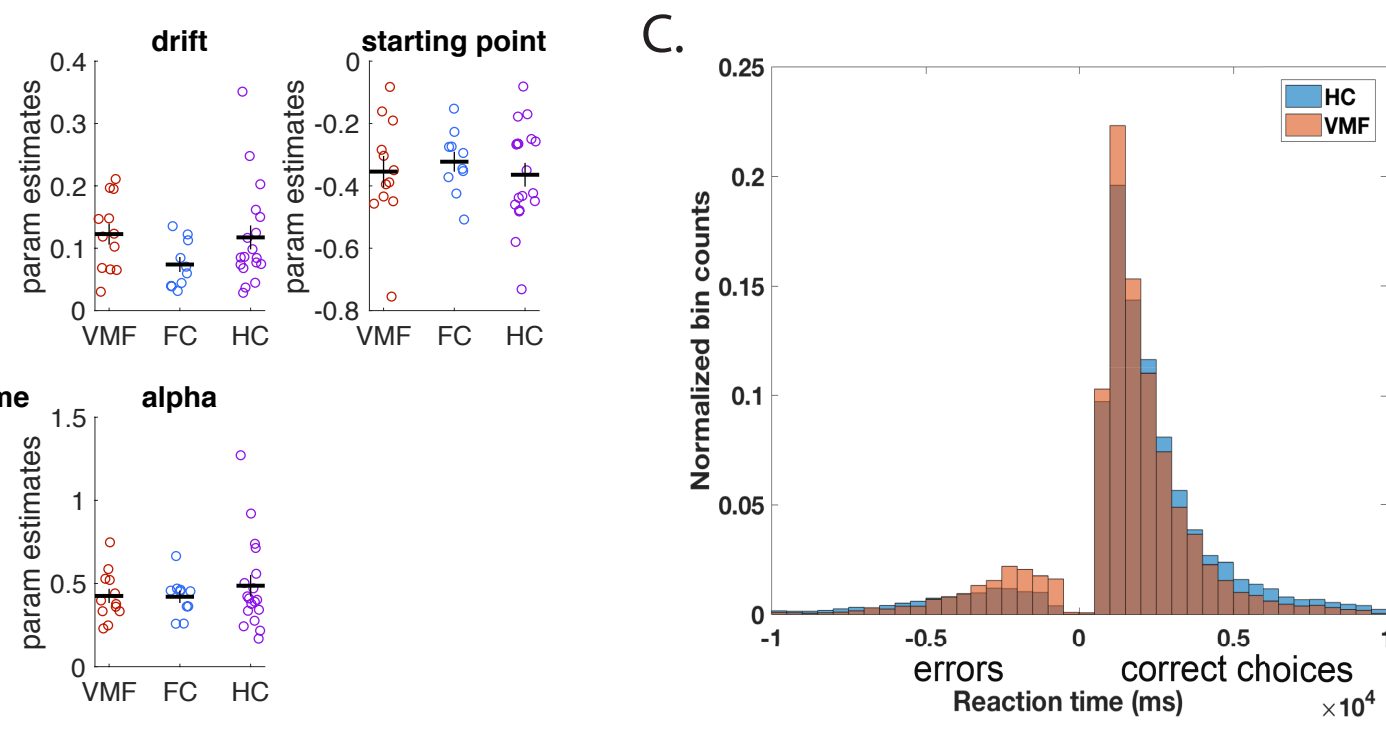

Figure 2. Participants with VMF lesions have higher decision noise. a) illustration of the drift diffusion model. After the non-decision time (part of the reaction time not related to the decision process), the decision variable begins at a starting value and accumulates evidence noisily at each time step at a certain drift rate $(d)$ towards either one of the two choice options, until it reaches threshold for one of them. b) DDM parameter fits: noise, drift rate, initial starting point, non-decision time, and alpha (exponent on rank distance). Error bars are standard errors of the mean. * denotes $p<0.05, * * *$ denotes $p<0.001 . \mathrm{N}=40$ individuals. b) Histogram of reaction times of all choices by HC group (orange) and VMF group (blue). RTs of "correct" choices, choosing the option with the higher average rank, are on the right, and RTs of "errors", choosing 
the option with the lower average rank, are mirrored on the left. $\mathrm{N}=4500$ data points (from 30 individuals).

\section{Individuals with frontal damage exhibit more choice cycles}

Second, in addition to showing that the estimated preference variability is larger in individuals with VMF damage, we also sought to directly replicate in our participants the previously documented tendency of individuals with VMF damage to exhibit more choice cycles. Such a replication would further support the conclusion that the preferences of individuals with VMF damage in our study are more variable. To do this, we examined the second subset of the choices in our experiment, Set B, which consisted of a single instance of all pairwise choices between a total of nine or ten items in each category. This allowed us to directly replicate two previous studies that counted the number of choice cycles (instances where a participant chose A over B, B over C, and C over A) in similarly designed choice sets, and found a greater number of choice cycles in individuals with VMF damage $(11,12)$.

We replicated the finding that individuals with VMF damage have more choice cycles than HC individuals (Figure 3). We first conducted a 2-way mixed measures ANOVA of the number of choice cycles made by each participant, with item Category (art, brands, gambles) as a within-subject factor and Group (VMF, FC, or $\mathrm{HC}$ ) as a between-subjects factor. We found a significant main effect of Group $[\mathrm{F}(1,41)=6.50, p=0.015]$, but no main effect of Category $(p=$ $0.57)$ nor a Group x Category interaction $(p=0.80)$. Then, we conducted a follow-up planned comparison between groups, combined across all three Categories. Similar to previous studies, our VMF group $($ mean $=9.93 \%, \mathrm{sd}=6.65)$ made more cyclical choices than the HC group (mean $=5.71 \%, \mathrm{sd}=4.05 ;$ Wilcoxon ranked sums $\mathrm{Z}=1.64, p=0.05)$. The percentage of cyclical 
choices we observed were also roughly similar to those previously reported for these two groups $(11,12)$.

However, we did not replicate that this increase in cyclical choices is selective to VMF damage in the frontal lobe. Unlike previous studies, our FC group $($ mean $=9.09 \%, \mathrm{sd}=3.74)$ also made more cyclical choices than the healthy control group $(Z=2.05, p=0.02)$ and the difference between VMF and FC group and was not significant $(Z=0.12, p=0.45)$. This increase in cyclical choices in the FC group was unexpected, and we consider possible interpretations of this result in the discussion.

In addition to testing whether individuals with VMF damage exhibited more choice cycles as a group, we also examined the number of cyclical choices at the individual level. We did this to establish that our conclusions applied not only at the group level for individuals with VMF damage as whole, but also at the individual level for at least some specific individuals within that group (especially as the test of stochastic transitivity is also performed at the level of individuals). To do this, we considered each individual with a VMF or other frontal lesion as a single case, and compared their percentage of choice cycles (across all three categories) against healthy controls. We made this comparison using case-control $t$-tests (19) which are modified to compare an individual against a normative group when the sample size is small. In the VMF group, four individuals made significantly more cyclical choices than healthy controls, before corrections for multiple comparisons (Subject 350: $\mathrm{t}(19)=2.04, p=0.03$; Subject 10403: $\mathrm{t}(19)=$ 3.28, $p=0.002$, Subject 12402: $\mathrm{t}(19)=3.13, p=0.003$; Subject 775: $\mathrm{t}(19)=3.13, p=0.003)$. These differences remained significant in the latter three individuals after correcting for multiple comparisons using FDR (corrected $p=0.023$ for all three individuals). As described in the supplement, we did not find that these three individuals differed from others in the VMF group 
in lesion location, lesion volume, or on any demographic variables. In contrast to the VMF group, none of the individuals in the FC group made significantly more cyclical choices than HC individualls (all $p>=0.05$ before multiple comparison correction).

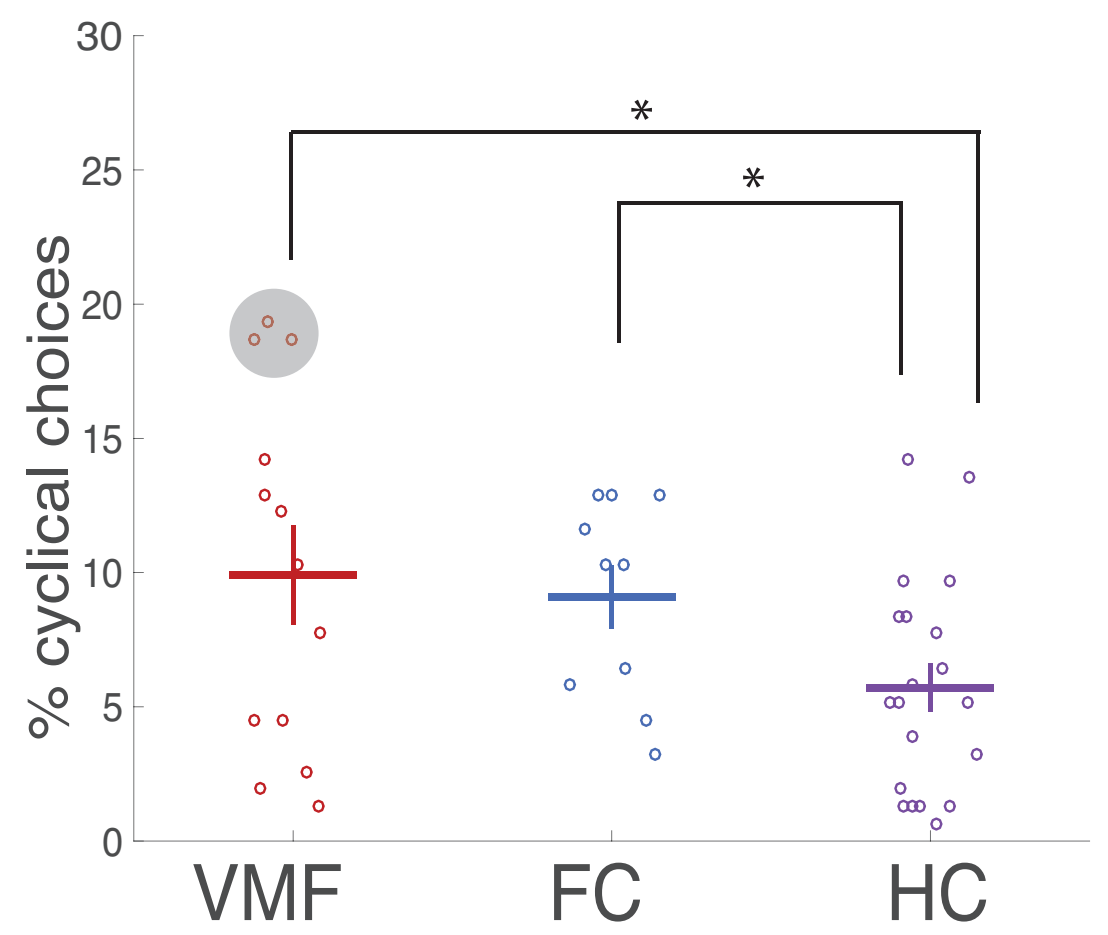

Figure 3. Individuals with frontal damage exhibit more choice cycles. Percentage of choice cycles across all domains at the group and individual levels. Dots encircled in gray denote the three individuals with VMF damage who made significantly more cyclical choices than healthy controls. Error bars are standard errors of the mean. * denotes $p<0.05 . \mathrm{N}=43$ individuals.

\section{Discussion}

Past demonstrations that individuals with VMF damage more frequently make cyclical choices (i.e., selecting A over B, B over C, and C over A; (11-13) cannot distinguish between two possibilities, with drastically different implications for the function of the VMF. One 
possibility is that individuals with VMF damage have preferences that are fundamentally intransitive. A second possibility is that individuals with VMF damage have preferences that are more variable, yet still fundamentally transitive. Here we provide a clear test between these two possibilities by evaluating whether the choices of individuals with VMF damage satisfy a stochastic model of transitivity. This model assumes that choices are always generated according to a transitive preference ordering, but allow the specific ordering to vary from choice to choice. Choosers with variable preferences fit this model, but choosers with intransitive preferences do not. We find unambiguous evidence that individuals with VMF damage do not have fundamentally intransitive preferences, even though their preferences are more variable, as all individuals with VMF damage make choices in all domains that are consistent with stochastic models of transitivity.

Not a single one of the individuals with VMF damage in our study violated the mixture model, a stochastic model of transitivity developed by Regenwetter et al. (14), even as their decisions, both as a group and for specific individuals, were more likely to exhibit choice cycles. We interpret this pattern of results to mean that individuals with VMF damage have preferences that are more variable. That is, as suggested by Henri-Bhargava, Simioni and Fellows (12), "values are unstable, fluctuating from trial to trial in those with VMF damage." We provide further support for this claim by fitting a drift diffusion model (18) to each individual's choices. In this model, the VMF group had a significantly higher noise term, i.e., more variability in the decision variable, than healthy individuals or those with frontal damage outside the VMF. Importantly, the VMF group did not differ from others in any other parameter of the DDM, showing that preference variability was the only dimension along which the VMF group differed. 
These results, particularly that individuals with VMF damage have higher decision noise in the DDM, are broadly consistent with previous studies that have linked choice variability to variability in neural value signals in VMF (20-22) and that have shown that disruptions of VMF cause higher choice variability $(23,24)$. Many of these studies have modelled variability as arising from random noise in underlying values, as in the DDM $(20,25)$, and thus have assumed a stochastic model of transitivity. However, such valuation models are not the only potential account of choice $(15,26,27)$. Our study takes a step beyond previous work by demonstrating that such valuation models are merited in our data, using an axiomatic test of a particular stochastic model of transitivity (28), the mixture model. The choices of all our subjects in the art and chocolate categories, and nearly all of our subjects in the gamble category, were consistent with the mixture model. Combined with previous work testing this model (14), this suggests that human choices in wide variety of domains may be consistent with such stochastic models of transitivity.

These results are difficult to reconcile with the view that the VMF is the only critical substrate for value-based choice (29). This view would predict that individuals with VMF damage would only be able to choose in a non-value-based manner, for example, according to rules or heuristics. Rules and heuristics can approximate transitive preference orderings under some conditions (30), but are not generally guaranteed to do so (15). In contrast, these results are easier to reconcile with a framework in which valuation and value-based choice are distributed processes, to which multiple regions of the brain contribute in some respect (31). This framework would predict that other regions can compensate for damage to the VMF, so that VMF damage does not fundamentally abolish the transitivity of preferences. As making transitive choices that maximize value is incredibly important to the survival of an organism, it 
would make sense that valuation is a highly conserved process that is robust to damage to one part of the cortex. Future studies could use functional brain imaging to more directly test hypotheses about compensation in individuals with VMF damage, as it is also possible that compensation occurs in the still intact parts of VMF rather than in other regions entirely.

Future studies can also further investigate how exactly the VMF supports the stability and reduces the variability of preferences. One possibility is that VMF contributes to the computation of subjective value. If subjective value is computed through the interaction of several brain regions, the loss of VMF may make this computation noisier and less reliable. An alternative possibility, though, is that the VMF contributes to the same preference ordering being repeated reliably, without contributing to valuation per se. For example, individuals might use episodic memories of their previous choices (e.g., "I remember choosing A over B before") to guide their decisions, or a representation of the context of the experiment may activate a specific set of preferences, as in a schematic network. Previous work has shown VMF involvement in both episodic memory processes $(32)$ and schema formation $(33,34)$.

Unexpectedly, we also found that the frontal control group made more cyclical choices than healthy controls. This finding is inconsistent with previous studies, where an increase in cyclical choices was specific to VMF damage in the frontal lobe. One difference between our study and previous ones is that ours included the choice category of gambles. As both the dorsomedial frontal, dorsolateral frontal, and insular cortices have been previously implicated in decisions about risk $(35,36)$, the inclusion of choices between gambles could account for the discrepancy in results. Future studies should examine the generality and replicability of our findings regarding other frontal regions. We hesitate to draw strong conclusions about the role of these other frontal regions in preference variability based on this result alone, however. First, 
unlike individuals with VMF damage, the frontal controls only made significantly more cyclical choices at the group level; none of the individuals in the frontal control group made significantly more cyclical choices at the individual level when compared to healthy controls in a case-control test. Second, unlike individuals with VMF damage, the frontal controls did not differ from healthy controls in decision noise, or any other parameter, when their choices were fit to a drift diffusion model. Thus, the differences in the frontal control group were only observed in one of the two choice sets in our experiment (Set B), whereas the differences in the VMF group were observed in both choice sets (Sets A and B). Nonetheless, to the extent that damage to frontal regions outside the VMF also increases preference variability, this would only further support the view that valuation is a distributed process in the brain.

In conclusion, we provided a clear-cut test of whether VMF is necessary for transitive preference and rational choice, and found that individuals with VMF damage have preferences that are more variable, but without exception, are fundamentally transitive. This result clarifies how erratic choices manifest after damage to the $\operatorname{VMF}(37,38)$ and potentially explains why studies using similar decision-making paradigms in individuals with VMF damage can yield different results (39). Our findings further characterize the necessary role the VMF plays in value-based decision-making. Specifically, though each choice still reflects a subjective preference ordering after VMF damage, an intact VMF is necessary for these preference orderings to remain stable and reliable across time and contexts.

\section{Methods}

\section{Experimental Design}


Participants. Fourteen individuals with focal damage to the frontal lobes were recruited from the Focal Lesion Database (FoLD) at the University of Pennsylvania, and ten were recruited from the Cognitive Neuroscience Research Registry at McGill University (40). Individuals were eligible to participate if they had a lesion primarily affecting the frontal lobes. One individual was excluded due to incomplete data collection (the individual completed one session and was not able to be scheduled for the second). Fourteen females and 9 males were included in the final sample. Participants were tested a minimum of 5 months after injury (median $=10.29$ years, range: 5 months to 17.75 years).

Participants were divided into two groups a priori based on location of damage, assessed with MR or computed tomography images by a neurologist blind to task performance. The VMF group consisted of individuals who sustained damage to the VMF, which is defined as the medial wall below the genu of the corpus callosum, and the orbitofrontal cortices. The FC group consisted of individuals who sustained damage to the frontal lobe sparing the VMF, which includes damage to dorsolateral, dorsomedial, and insular cortices. Lesions were drawn on a common space [Montreal Neurological Institute (MNI) brain] by neurologists at the research sites blind to task performance. The overlap images for the groups are presented in Figure 1. Damage in the VMF group was caused by aneurysm or subarachnoid hemorrhage in 5 cases, stroke in 2 cases, tumor resection in 3 cases, glioma in one case, and meningioma in 2 cases. Damage in the FC group was caused by hemorrhage, stroke or infarct in 7 cases, glioma in 2 cases, and meningioma in one case.

Age and education matched $\mathrm{HC}$ individuals were recruited from the corresponding Normal Control Databases of the University of Pennsylvania $(\mathrm{N}=14)$ and McGill University $(\mathrm{N}$ $=6$ ), including 15 females and 5 males (Table 1). They were free of neurological and psychiatric 
disorders. All subjects provided informed consent and were compensated for their time. The study protocol was approved by the institutional review boards of both the University of Pennsylvania and McGill University.

Apparatus. All tasks were programmed using EPrime 2.0 (Psychology Software Tools). Participants were tested at the Hospital of the University of Pennsylvania, at the MNI, or at their own home in the greater Philadelphia or Montreal area. Participants saw stimuli on a laptop monitor and responded using the 1 and 0 keys of the keyboard.

Items. Choice items consisted of images of artwork, chocolate bars that differed in brand, and gambles (presented as pie charts). There were two sets of items: 5 items from each of the categories (art, chocolate bar brands, gambles) that were used in repeated choices that allow stochastic tests of transitivity (set A); and 10-11 items from each of the categories (10 for chocolate bar brands, 11 for art and gambles) used in non-repeated choices that allow testing for choice cycles (set B). Choices constructed using set A and set B stimuli were intermingled in each block. For both the art and chocolate bar brand categories, we designed item sets in which the items were normed to be close in preference. The gambles in the gamble categories were all of equal expected value (Supplementary methods).

Procedure. Participants completed a binary forced choice task. On each trial, participants first saw a central fixation point for 1s, then a screen with two choice items (placed to the left and the right of the center). Participants indicated which item they preferred, by pressing buttons for left or right. Participants had as much time as they needed to make their selection. Following their selection, there was an inter-trial interval of $1 \mathrm{~s}$ where a black screen was presented.

For set A stimuli, participants faced all possible pairings of 5 items, constituting 10 pairs, and each pair was repeated 15 times. For set B stimuli, participants faced all possible pairings of 
either 10 (for brands) or 11 (for art and gambles) items, constituting 45 or 55 pairs in total, respectively, and each pair was presented once. Therefore, there were 195 (for brands) or 205 (for art and gambles) total choices in each category across the entire experiment.

Choice trials were presented in blocks, in which participants made choices between items within a single category (art, brands, gambles). There were five blocks of choices for each category, containing 39 (for brands) or 41 (for art and gambles) trials each. Each block contained 30 choices composed from set A and 9 or 11 choices composed from set B. Choices from set A and set $\mathrm{B}$ were intermingled with each other within a block, with the set B choices inserted into a block of A choices in positions randomly selected from a uniform distribution.

We took several steps to reduce any potential memory effects for repeated set A choices. We designed the sequence of trials so that: (1) the same pairing was not repeated within a minimum of 3 trials; (2) the same item rarely appeared on immediately adjacent trials (no more than 9 times throughout the entire experiment); and (3) when the same pairing was repeated the choices immediately preceding and following that pairing differed from its previous occurrence (to minimize contextual memory). Furthermore, the side on which stimuli were presented was counterbalanced across repetitions. Finally, we divided the experiment into two sessions, held on separate days for every subject except two (due to scheduling constraints). The two sessions were held on average $8.09(\mathrm{sd}=11.73)$ days apart (excepting the two who were tested on the same day, the sessions ranged from 1 day to 57 days apart). We did not observe a significant correlation between the total number of choice cycles and days between the two sessions $(\mathrm{r}=$ $0.24, p=0.12)$

\section{Statistical Analysis}




\section{Tests of a stochastic model of transitivity. All data were analyzed with MATLAB}

(Mathworks). We used the Set A choices to perform tests of a stochastic model of transitivity. We first obtained the choice percentages (out of a possible total of 15 choices) for each of the 10 choice pairs (representing all possible pairings of the 5 items) in each category. We then tested the mixture model of preference described by Regenwetter and colleagues (14). The mixture model states that a person's response comes from a probability distribution over all possible orderings of the items. Thus, at any one time, preferences are transitive, but the transitive state that one is in can vary. The probability of a person choosing one item (X) over another (Y) in a binary choice is the sum of all the preference states in which $\mathrm{X}$ is preferred to $\mathrm{Y}$. In a two alternative forced choice task, this probability is constrained by the triangle inequalities. For every distinct $\mathrm{X}, \mathrm{Y}$, and $\mathrm{Z}$ in a choice set:

$$
\mathrm{P}_{\mathrm{xy}}+\mathrm{P}_{\mathrm{yz}}-\mathrm{P}_{\mathrm{xz}} \leq 1
$$

Where $\mathrm{P}_{\mathrm{xy}}$ denotes the probability of choosing $\mathrm{X}$ over $\mathrm{Y}$, etc. For up to 5 options in a two alternative forced choice task, satisfying the triangle inequalities is necessary and sufficient for a set of choices to be consistent with the mixture model.

For choice percentages that did not satisfy the triangle inequalities, we used the Q-test (41) software to determine whether these violations were unlikely to be due to random sampling. Q-test uses maximum likelihood estimation to find the goodness of fit of the data at each vertex in the linear ordering polytope defined by the triangle inequalities, using a chi-squared bar distribution with simulated weights $(41,42)$. A Q-test with $p<0.05$ was taken as evidence that a subject's choices in that category were inconsistent with the mixture model of preference.

Drift diffusion modelling and analysis of reaction times. We fit a drift diffusion model (18) to the choices and RTs from all set B choices for every subject and category in our 
experiment. We modelled the decision process as a decision variable (DV) that increased linearly with a slope $d^{*} v^{\alpha}$, where $d$ was the drift rate, $v$ was the value difference between the items (expressed as the absolute rank difference between the two items for that individual), and $\alpha$ was an exponent accounting for potential non-linearities in the effect of rank difference. We also assumed that at each time step there is Gaussian noise added to the DV, with a standard deviation of $\varepsilon$. We assumed $10 \mathrm{~ms}$ time steps. We also assumed there is a non-decision time $(n d t)$ before accumulation begins, and an initial value (int) of the DV that is constant across trials. Choices are made when the DV crosses a threshold.

Thus there are five free parameters: $d, \alpha, \varepsilon, i n t$ and $n d t$. Note that the threshold was a fixed parameter across subjects, as one of the threshold, $d$, or $\varepsilon$ must be fixed for the other two parameters to be estimable. We chose to fix threshold after a model-comparison process showed that option to provide the best model fits. Threshold was held constant at (+/-) 0.15 . Values for $d$ are sampled between 0 and 1 , for $\varepsilon$ are sampled between 0 and 1 , for $\alpha$ are sampled between 0 and 3, for int are sampled between the threshold bounds, and for $n d t$ are sampled between 0 and the minimum RT minus $10 \mathrm{~ms}$ for that subject.

To fit these free parameters, we first calculated the cumulative probability that the DV crossed the threshold for the subject's choice ( $T_{\text {correct }}$ or $T_{\text {incorrect, }}$ where "correct" was defined as choosing the option of higher rank) across all time steps. For each trial, we then calculated the joint likelihood of the subject's choice at the time which they made that choice (their trial RT, minus $n d t$ ), by taking the derivative of this cumulative probability at the timestep of the subject's choice (every $10 \mathrm{~ms}$ to the maximum RT for the subject). The model was then fit using the MATLAB function fmincon, where the cost function was defined as the sum of the negative log likelihoods of the instantaneous probabilities of the subject's choices and RTs in all trials. The 
fitting procedure was repeated 10 times for each subject, with each iteration varying in randomly sampled starting values for the free parameters as specified above; the parameters with the lowest log likelihood out of the 10 was taken for that subject. The model was fit individually to each of the three categories (art, brands, gambles) for each subject.

We calculated the average ranks of the items according to the number of times each item was chosen, with the item that was chosen most often overall ranked first, the item chosen second-most ranked second, etc. We broke ties by looking at which item was more often chosen more than half of the time in every pair (12). Three subjects still had tied ranks after this process, in one category each: two were $\mathrm{HC}$ subjects in the gambles domain, and the other was a VMF subject in the Art domain. These subjects in these categories only were dropped from the drift diffusion modelling.

To look at differences in DDM parameters between groups across categories, we performed a mixed ANOVA on each of the free parameters, with group as the cross-subject factor and reward category as the within-subject factor.

Choice cycles. We used the set B choices to directly replicate previous studies that counted the number of choice cycles. We first determined the preference ordering within each category for each subject. The 10 or 11 options within each category were ranked according to how many times each was chosen by that subject. Then, for each trial, a choice was counted as cyclical if a lower-ranked item was chosen over a higher-ranked item. Following HenriBhargava, Simioni and Fellows (12), ties were maintained in the rankings (i.e., more than option could have the same rank) to provide a more conservative definition of cyclical choices. Because the cyclical choice counts are not normally distributed, we used non-parametric statistics to test for group differences. We used Kruskal-Wallis tests to detect effects between groups, followed 
by one-tailed Wilcoxon ranked sum post hoc pairwise tests when testing for effects between VMF and HC (as several previous studies have found increased cyclical choices after VMF damage, we had strong hypotheses about the direction of the results). To test for within-subject effects of choice category, we used repeated measures analysis of variance (ANOVA) on ranktransformed data for the omnibus test and Wilcoxon signed-rank post hoc tests as appropriate. 
Acknowledgments: We would like to thank Avinash Vaidya and Arthur Lee for constructive discussion on aspects of data analysis. We would also like to thank Lesley Fellows for facilitating access to participants in Montreal, Christine Déry and Eileen Cardillo for coordinating participants in Montreal and in Philadelphia, and all of the participants themselves, without whom this work would not be possible. This work was supported by National Institute on Drug Abuse (NIDA) R01-DA029149 to JWK and a Natural Sciences and Engineering Research Council (NSERC) postgraduate doctoral scholarship to LQY. QTEST was developed with support by the National Science Foundation grants SES 10-62045 and SES 14-59699 (PI: M. Regenwetter) as well as by the Humboldt Foundation (Co-PIs: J. Stevens and M. Regenwetter). 


\section{References}

1. P. A. Samuelson, A note on measurement of utility. The review of economic studies 4, 155-161 (1937).

2. O. Bartra, J. T. McGuire, J. W. Kable, The valuation system: a coordinate-based metaanalysis of BOLD fMRI experiments examining neural correlates of subjective value. Neuroimage 76, 412-427 (2013).

3. D. J. Levy, P. W. Glimcher, The root of all value: a neural common currency for choice. Current Opinion in Neurobiology 22, 1027-1038 (2012).

4. C. Padoa-Schioppa, J. A. Assad, Neurons in the orbitofrontal cortex encode economic value. Nature 441, 223-226 (2006).

5. C. Padoa-Schioppa, J. A. Assad, The representation of economic value in the orbitofrontal cortex is invariant for changes of menu. Nature neuroscience 11, 95-102 (2008).

6. L. Clark et al., Differential effects of insular and ventromedial prefrontal cortex lesions on risky decision-making. Brain: A Journal of Neurology 131, 1311-1322 (2008).

7. B. Studer, F. Manes, G. Humphreys, T. W. Robbins, L. Clark, Risk-sensitive decisionmaking in patients with posterior parietal and ventromedial prefrontal cortex injury. Cerebral cortex (New York, N.Y. : 1991) 25, 1-9 (2015).

8. L. K. Fellows, M. J. Farah, Dissociable elements of human foresight: A role for the ventromedial frontal lobes in framing the future, but not in discounting future rewards. Neuropsychologia 43, 1214-1221 (2005).

9. M. Sellitto, E. Ciaramelli, G. di Pellegrino, Myopic Discounting of Future Rewards after Medial Orbitofrontal Damage in Humans. The Journal of Neuroscience 30, 16429-16436 (2010).

10. J. Peters, M. D'Esposito, Effects of medial orbitofrontal cortex lesions on self-control in intertemporal choice. Current Biology 26, 2625-2628 (2016).

11. L. K. Fellows, M. J. Farah, The role of ventromedial prefrontal cortex in decision making: judgment under uncertainty or judgment per se? Cerebral cortex (New York, N.Y. : 1991) 17, 2669-2674 (2007).

12. A. Henri-Bhargava, A. Simioni, L. K. Fellows, Ventromedial frontal lobe damage disrupts the accuracy, but not the speed, of value-based preference judgments. Neuropsychologia 50, 1536-1542 (2012).

13. N. Camille, C. A. Griffiths, K. Vo, L. K. Fellows, J. W. Kable, Ventromedial frontal lobe damage disrupts value maximization in humans. The Journal of neuroscience : the official journal of the Society for Neuroscience 31, 7527-7532 (2011).

14. M. Regenwetter, J. Dana, C. P. Davis-Stober, Transitivity of preferences. Psychological review 118, 42-56 (2011).

15. A. Tversky, Intransitivity of preferences. Psychological review (1969).

16. D. McFadden, Econometric Models for Probabilistic Choice Among Products. The Journal of Business 53, S13-S29 (1980). 
17. R. Luce (1959) Individual Choice Behaviour. A Theorical Analysis. (New York. Willey).

18. R. Ratcliff, A theory of memory retrieval. Psychological review 85, 59 (1978).

19. J. R. Crawford, D. C. Howell, Comparing an individual's test score against norms derived from small samples. The Clinical Neuropsychologist 12, 482-486 (1998).

20. R. Webb, I. Levy, S. C. Lazzaro, R. B. Rutledge, P. W. Glimcher, Neural random utility: Relating cardinal neural observables to stochastic choice behavior. Journal of Neuroscience, Psychology, and Economics 12, 45 (2019).

21. V. Kurtz-David, D. Persitz, R. Webb, D. J. Levy, The neural computation of inconsistent choice behavior. Nature communications 10, 1-14 (2019).

22. C. Padoa-Schioppa, Neuronal origins of choice variability in economic decisions. Neuron 80, 1322-1336 (2013).

23. R. Polanía, M. Moisa, A. Opitz, M. Grueschow, C. C. Ruff, The precision of value-based choices depends causally on fronto-parietal phase coupling. Nature communications 6, 110 (2015).

24. S. Ballesta, W. Shi, K. E. Conen, C. Padoa-Schioppa, Values encoded in orbitofrontal cortex are causally related to economic choices. Nature 588, 450-453 (2020).

25. R. Webb, The (neural) dynamics of stochastic choice. Management Science 65, 230-255 (2019).

26. E. Brandstätter, G. Gigerenzer, R. Hertwig, The priority heuristic: making choices without trade-offs. Psychological review 113, 409 (2006).

27. B. Hayden, Y. Niv, The case against economic values in the brain. (2020).

28. R. B. Rutledge, M. Dean, A. Caplin, P. W. Glimcher, Testing the reward prediction error hypothesis with an axiomatic model. Journal of Neuroscience 30, 13525-13536 (2010).

29. C. Padoa-Schioppa, X. Cai, Orbitofrontal cortex and the computation of subjective value: consolidated concepts and new perspectives. Annals of the New York Academy of Sciences 1239, 130 (2011).

30. A. Rubinstein (1990) New directions in economic theory-Bounded rationality.

31. L. T. Hunt, B. Y. Hayden, A distributed, hierarchical and recurrent framework for reward-based choice. Nature Reviews Neuroscience 18, 172 (2017).

32. E. Bertossi, C. Tesini, A. Cappelli, E. Ciaramelli, Ventromedial prefrontal damage causes a pervasive impairment of episodic memory and future thinking. Neuropsychologia http://dx.doi.org/10.1016/j.neuropsychologia.2016.01.034 (2016).

33. M. L. Schlichting, A. R. Preston, Hippocampal-medial prefrontal circuit supports memory updating during learning and post-encoding rest. Neurobiology of learning and memory 134, 91-106 (2016).

34. K. N. Spalding et al., Ventromedial prefrontal cortex is necessary for normal associative inference and memory integration. Journal of Neuroscience 38, 3767-3775 (2018).

35. M. Hsu, I. Krajbich, C. Zhao, C. F. Camerer, Neural response to reward anticipation under risk is nonlinear in probabilities. Journal of Neuroscience 29, 2231-2237 (2009).

36. G. I. Christopoulos, P. N. Tobler, P. Bossaerts, R. J. Dolan, W. Schultz, Neural correlates of value, risk, and risk aversion contributing to decision making under risk. Journal of Neuroscience 29, 12574-12583 (2009).

37. J. M. Harlow, Recovery from the passage of an iron bar through the head. Publications of the Massachusetts Medical Society 2, 274-281 (1868). 
38. P. J. Eslinger, A. R. Damasio, Severe disturbance of higher cognition after bilateral frontal lobe ablation. Patient EVR 35, 1731-1731 (1985).

39. L. K. Fellows, 16 The Neurology of Value. Neurobiology of sensation and reward, 351 (2011).

40. L. K. Fellows, M. Stark, A. Berg, A. Chatterjee, Patient registries in cognitive neuroscience research: Advantages, challenges, and practical advice. Journal of Cognitive Neuroscience 20, 1107-1113 (2008).

41. M. Regenwetter et al., QTest: Quantitative testing of theories of binary choice. Decision 1, 2 (2014).

42. M. Regenwetter, J. Dana, C. P. Davis-Stober, Testing transitivity of preferences on twoalternative forced choice data. Frontiers in psychology 1, 148 (2010). 


\section{Tables}

Table 1: Demographics of participants

\begin{tabular}{lccc}
\hline $\begin{array}{l}\text { Group } \\
\text { (n) }\end{array}$ & Gender & Mean age (sd) & $\begin{array}{l}\text { Education in } \\
\text { yrs (sd) }\end{array}$ \\
\hline VMF (13) & $7 \mathrm{~F}: 6 \mathrm{M}$ & $59(15)$ & $14(3)$ \\
FC (10) & $7 \mathrm{~F}: 3 \mathrm{M}$ & $66(8)$ & $14(3)$ \\
$\mathrm{HC} \mathrm{(20)}$ & $15 \mathrm{~F}: 5 \mathrm{M}$ & $62(8)$ & $15(2)$ \\
\hline
\end{tabular}

Table 2: Choice percentages (in Set A) for four individuals with VMF damage

The complete set of choice percentages in each stimulus category in Set A, for each of four individuals with VMF damage. These are the same four individuals who had significantly more choices cycles than healthy controls in Figure 3. The options are ranked A-E, where A is the option that was chosen most often by that subject, B is the option chosen second most often, etc., and the numbers are the percentage of choices where the column option is chosen over the row option. 


\begin{tabular}{|c|c|c|c|c|c|c|c|c|c|c|c|c|c|c|c|c|c|c|}
\hline \multirow[b]{3}{*}{ VMF2 } & \multicolumn{18}{|c|}{ Category } \\
\hline & \multicolumn{6}{|c|}{ Art } & \multicolumn{6}{|c|}{ Brands } & \multicolumn{6}{|c|}{ Gamble } \\
\hline & & $\mathbf{A}$ & B & $\mathrm{C}$ & D & $\mathbf{E}$ & & $\mathbf{A}$ & B & $\mathrm{C}$ & D & $\mathbf{E}$ & & $\mathbf{A}$ & B & $\mathrm{C}$ & D & $\mathbf{E}$ \\
\hline & A & & & & & & A & & & & & & A & & & & & \\
\hline & B & 93 & & & & & B & 67 & & & & & B & 100 & & & & \\
\hline & $\mathrm{C}$ & 100 & 67 & & & & $\mathbf{C}$ & 60 & 40 & & & & $\mathrm{C}$ & 87 & 93 & & & \\
\hline & D & 100 & 80 & 33 & & & D & 73 & 87 & 60 & & & D & 100 & 100 & 73 & & \\
\hline & $\mathbf{E}$ & 93 & 67 & 80 & 47 & & $\mathbf{E}$ & 80 & 87 & 73 & 67 & & $\mathbf{E}$ & 93 & 100 & 100 & 87 & \\
\hline VMF8 & & $\mathbf{A}$ & B & $\mathrm{C}$ & D & $\mathbf{E}$ & & $\mathbf{A}$ & B & $\mathrm{C}$ & D & $\mathbf{E}$ & & $\mathbf{A}$ & B & $\mathrm{C}$ & D & $\mathbf{E}$ \\
\hline & $\mathbf{A}$ & & & & & & $\mathbf{A}$ & & & & & & $\mathbf{A}$ & & & & & \\
\hline & B & 67 & & & & & B & 60 & & & & & B & 33 & & & & \\
\hline & $\mathrm{C}$ & 80 & 40 & & & & $\mathrm{C}$ & 93 & 80 & & & & $\mathrm{C}$ & 67 & 47 & & & \\
\hline & D & 100 & 100 & 80 & & & D & 73 & 67 & 80 & & & D & 67 & 67 & 47 & & \\
\hline & $\mathbf{E}$ & 100 & 93 & 100 & 67 & & $\mathbf{E}$ & 80 & 93 & 80 & 40 & & $\mathbf{E}$ & 80 & 53 & 80 & 67 & \\
\hline VMF10 & & $\mathbf{A}$ & B & C & D & $\mathbf{E}$ & & $\mathbf{A}$ & B & $\mathrm{C}$ & D & $\mathbf{E}$ & & $\mathbf{A}$ & B & C & D & $\mathbf{E}$ \\
\hline & $\mathbf{A}$ & & & & & & $\mathbf{A}$ & & & & & & $\mathbf{A}$ & & & & & \\
\hline & B & 100 & & & & & B & 100 & & & & & B & 93 & & & & \\
\hline & $\mathbf{C}$ & 100 & 100 & & & & $\mathrm{C}$ & 100 & 100 & & & & $\mathrm{C}$ & 100 & 93 & & & \\
\hline & D & 100 & 100 & 93 & & & D & 100 & 100 & 100 & & & D & 93 & 87 & 93 & & \\
\hline & $\mathbf{E}$ & 100 & 100 & 100 & 100 & & $\mathbf{E}$ & 100 & 100 & 100 & 87 & & $\mathbf{E}$ & 100 & 100 & 93 & 93 & \\
\hline VMF12 & & $\mathbf{A}$ & B & $\mathrm{C}$ & D & $\mathbf{E}$ & & $\mathbf{A}$ & B & $\mathrm{C}$ & D & $\mathbf{E}$ & & $\mathbf{A}$ & B & C & D & $\mathbf{E}$ \\
\hline & $\mathbf{A}$ & & & & & & A & & & & & & A & & & & & \\
\hline & B & 60 & & & & & B & 60 & & & & & B & 40 & & & & \\
\hline & $\mathbf{C}$ & 53 & 53 & & & & $\mathbf{C}$ & 60 & 47 & & & & $\mathbf{C}$ & 87 & 73 & & & \\
\hline & D & 67 & 73 & 67 & & & D & 60 & 80 & 47 & & & D & 73 & 67 & 73 & & \\
\hline & $\mathbf{E}$ & 60 & 67 & 73 & 53 & & $\mathbf{E}$ & 67 & 53 & 67 & 60 & & $\mathbf{E}$ & 93 & 67 & 73 & 60 & \\
\hline
\end{tabular}




\section{Table 3: Results of LOP analysis, by category}

Art Brands Gambles

$\frac{\text { Respondent }}{\text { Individuals with VMF lesions }}$

Individuals with VMF lesions p-value p-value p-value

Frontal controls

$\begin{aligned} 1 & \checkmark \\ 2 & \checkmark \\ 3 & \checkmark \\ 4 & \checkmark \\ 5 & \checkmark \\ 6 & \checkmark \\ 7 & \checkmark \\ 8 & \checkmark \\ 9 & \checkmark \\ 10 & \checkmark \\ 11 & \checkmark \\ 12 & \checkmark \\ 13 & \checkmark\end{aligned}$

$\begin{array}{cc}\checkmark & \checkmark \\ \checkmark & 0.64 \\ \checkmark & 0.83 \\ \checkmark & \checkmark \\ \checkmark & \checkmark \\ \checkmark & \checkmark \\ 0.57 & \checkmark \\ \checkmark & \checkmark \\ \checkmark & 0.92 \\ \checkmark & \checkmark \\ \checkmark & \checkmark \\ \checkmark & \checkmark \\ \checkmark & \checkmark\end{array}$

Frontal controls

$\begin{array}{rl}1 & 0.2 \\ 2 & \checkmark \\ 3 & \checkmark \\ 4 & \checkmark \\ 5 & \checkmark \\ 6 & \checkmark \\ 7 & \checkmark \\ 8 & \checkmark \\ 9 & \checkmark \\ 10 & \checkmark\end{array}$

Healthy controls

$\begin{array}{ll}1 & \checkmark \\ 2 & \checkmark \\ 3 & \checkmark \\ 4 & \checkmark \\ 5 & \checkmark \\ 6 & \checkmark \\ 7 & \checkmark \\ 8 & \checkmark \\ 9 & \checkmark\end{array}$

$\begin{array}{cc}0.71 & \mathbf{0 . 0 0 1 6} \\ \checkmark & 0.9 \\ \checkmark & \checkmark \\ \checkmark & \checkmark \\ \checkmark & \checkmark \\ \checkmark & \checkmark \\ \checkmark & \checkmark \\ \checkmark & 0.95 \\ \checkmark & \mathbf{0 . 0 1}\end{array}$




$\begin{array}{lc}10 & \checkmark \\ 11 & \checkmark \\ 12 & \checkmark \\ 13 & \checkmark \\ 14 & \checkmark \\ 15 & 0.24 \\ 16 & \checkmark \\ 17 & \checkmark \\ 18 & \checkmark \\ 19 & \checkmark \\ 20 & \checkmark\end{array}$

$\begin{array}{cc}\checkmark & 0.55 \\ \checkmark & \checkmark \\ \checkmark & 0.09 \\ \checkmark & \checkmark \\ \checkmark & \checkmark \\ \checkmark & \checkmark \\ 0.87 & 0.27 \\ \checkmark & \checkmark \\ 0.36 & \checkmark \\ \checkmark & 0.26 \\ \checkmark & \checkmark\end{array}$

Note: Each participant participated in choices for all three categories.

Checkmark indicates subject fulfilled triangle inequalities for that category. Significant violations of linear ordering polytope are marked in bold. 